\section{Obesidade na adolescência \\ e seus principais fatores determinantes}

\section{Obesity in adolescence and its main determinants}

\section{Carla Cristina Enes}

\section{Betzabeth Slater}

Departamento de Nutrição da Faculdade de Saúde Pública da Universidade de São Paulo

Enes CC participou da revisão de literatura, análise crítica dos artigos e redação do manuscrito Slater B participou da redação e revisão final do manuscrito.

Declaração de conflito de interesse: nada a declarar.

Correspondência: Carla Cristina Enes. Rua Capitão Alfredo Cardoso, 54 Jardim Faculdade, Sorocaba, SP CEP 18030-310. E-mail: cenes@usp.br

\section{Resumo}

O objetivo deste trabalho foi discutir os principais fatores ambientais determinantes do sobrepeso e da obesidade em adolescentes, fundamentando-se em uma revisão crítica sobre o assunto. Para a revisão do tema foram consultadas as principais bases de dados nacionais e internacionais, entre elas, Medline/Pubmed, Web of Science, SciELO, Lilacs, compreendendo o período de 1975 a 2009. Os descritores e termos MeSH utilizados na busca foram: "sobrepeso", "obesidade”, "adolescência”, “adolescentes”, "atividade física”, "ingestão alimentar”. Os resultados das pesquisas indicam que as mudanças ocorridas nos padrões alimentares nas últimas décadas, como o aumento do consumo de açúcares simples, alimentos industrializados e ingestão insuficiente de frutas e hortaliças, estão diretamente associadas ao ganho de peso dos adolescentes. Além disso, a redução progressiva da prática de atividade física combinada ao maior tempo dedicado às atividades de baixa intensidade, como assistir televisão, usar computador e jogar videogame, também tem contribuído para o aumento de peso dos jovens. Conclui-se, portanto, que as variáveis relacionadas ao padrão alimentar e de atividade física devem ser priorizadas nas intervenções voltadas para a prevenção da obesidade entre adolescentes.

Palavras-chave: Adolescente. Alimentação. Atividade física. Obesidade. Sobrepeso 


\section{Abstract}

The objective of this paper was to discuss the main environmental factors determining overweight and obesity in adolescents, based on a critical review of the subject. The main national and international health databases, Medline/PubMed, Web of Science, SciELO, and Lilacs were searched including publications from 1975 to 2009. The following key-words and respective MeSH terms were used: "overweight”, "obesity”, "adolescence”, "adolescents", "physical activity", "food intake". The findings showed that changes in dietary patterns in recent decades as the increased consumption of simple sugars, processed foods, and inadequate intake of fruits and vegetables have contributed directly to the weight gain in this population. In addition, the progressive reduction in physical activity associated with increased time spent with low-intensity activities like television viewing, playing computer and video games has contributed to the weight gain of adolescents. In conclusion, variables related to dietary pattern and physical activity should be prioritized interventions directed toward the prevention of obesity among adolescents.

Keywords: Adolescent. Feeding. Physical activity. Obesity. Overweight.

\section{Introdução}

A obesidade tem sido descrita como um importante problema de saúde pública da atualidade e vem ganhando destaque no cenário epidemiológico mundial. Sua prevalência aumentou nas últimas décadas em todo o mundo, inclusive nos países em desenvolvimento, como o Brasil, onde anteriormente predominavam os problemas relacionados à desnutrição ${ }^{1}$.

O aumento da prevalência de sobrepeso e obesidade em idades cada vez mais precoces tem despertado a preocupação de pesquisadores e profissionais da área de saúde, em razão dos danos e agravos à saúde provocados pelo excesso de peso, tais como hipertensão arterial, cardiopatias, diabetes, hiperlipidemias, dentre outras ${ }^{2,3}$.

Em 2004, a estimativa de excesso de peso entre crianças e adolescentes era de $10 \%$; destes, 25\% eram jovens obesos ${ }^{4}$. Estudo realizado porWang et al. ${ }^{5}$ em países com diferentes estágios de desenvolvimento socioeconômico revelou aumento significativo na prevalência de sobrepeso entre crianças e adolescentes nas últimas décadas. Entre os adolescentes, foram observados incrementos de magnitude importante: $62 \%$ nos Estados Unidos (de 16,8\% para 27,3\%) e $240 \%$ no Brasil (de 3,7\% para $12,6 \%$ ).

No período da adolescência, além das transformações fisiológicas, o indivíduo sofre importantes mudanças psicossociais, o que contribui para a vulnerabilidade característica desse grupo populacional. Os adolescentes podem ser considerados um grupo de risco nutricional, devido à inadequação de sua dieta decorrente do aumento das necessidades energéticas $\mathrm{e}$ de nutrientes para atender à demanda do crescimento.

Feijó et al. ${ }^{6}$ chamam a atenção para a importância da consolidação de hábitos alimentares e de estilo de vida saudáveis na adolescência. Segundo os autores, é nessa fase que esses hábitos são estabelecidos e muitas vezes mantidos na vida adulta.

Considerando que a adolescência é um período da vida no qual ocorrem intensas mudanças físicas e psicológicas, influenciadas por fatores genéticos, étnicos e ainda pelas diferentes condições sociais e ambientais, o objetivo deste trabalho foi discutir os principais determinantes ambientais do sobrepeso e da obesidade entre os adolescentes.

\section{Prevalência de sobrepeso e obesidade}

Estudos nacionais, especialmente de base populacional, que investigaram a prevalência de sobrepeso e obesidade no Brasil são escassos. Os dados disponíveis atualmente foram obtidos por meio do Estudo Nacional da Despesa Familiar (ENDEF), rea- 
lizado na década de 70, Pesquisa Nacional sobre Saúde e Nutrição (PNSN), realizada em 1989 e Pesquisa sobre Padrões de Vida (PPV) realizada em meados da década de 90 nas regiões Nordeste e Sudeste do Brasil.

Estudos mais recentes nesta área geralmente têm uma abrangência regional. Além disso, há uma grande variabilidade nas taxas de prevalência de sobrepeso e obesidade identificadas nessas pesquisas. Tais discrepâncias podem estar relacionadas às diferentes faixas etárias investigadas e à adoção de parâmetros distintos para classificação do estado nutricional.

Em um estudo de base populacional realizado em Pelotas-RS, Neutzling et al. ${ }^{7}$ encontraram prevalência de obesidade de $10,6 \%$ entre as meninas e $4,8 \%$ entre os meninos, utilizando o critério de classificação proposto pela Organização Mundial de Saúde (excesso de peso: $\geq$ percentil 85 e obesidade: $\geq$ percentil 95).

Terres et al. ${ }^{8}$, em um estudo transversal de base populacional realizado também no município de Pelotas-RS, investigaram a prevalência de sobrepeso e obesidade entre adolescentes de 15 a 18 anos. Os autores identificaram prevalência de $20,9 \%$ para sobrepeso e 5,0\% para obesidade. O critério utilizado para a classificação do estado nutricional foi o proposto por Cole et al. ${ }^{9}$, recomendado pela International Obesity Task Force - IOTF, baseados nos valores de desfecho de sobrepeso (valores equivalentes a IMC maior que $25 \mathrm{~kg} / \mathrm{m}^{2}$ e menor que $30 \mathrm{~kg} / \mathrm{m}^{2} \mathrm{em}$ adultos) e obesidade (valores equivalentes ao IMC igual ou maior que 30 $\mathrm{kg} / \mathrm{m}^{2} \mathrm{em}$ adultos).

A partir de um estudo transversal com 10.822 crianças de 7 a 10 anos realizado no município de Santos, Costa et al. ${ }^{10}$ encontraram prevalência de sobrepeso e obesidade de $15,7 \%$ e $18 \%$, respectivamente, entre os jovens avaliados.

Farias et al. ${ }^{11}$, a partir de um estudo realizado com 1.057 jovens da mesma faixa etária (7 a 10 anos), observaram prevalências menores de sobrepeso $(7,0 \%)$ e obesidade $(3,0 \%)$. O critério de classificação do estado nutricional baseou-se nas curvas do Centers for Disease Control and Prevention (NCHS), assim como no estudo de Costa et al. ${ }^{10}$.

Ricardo et al. ${ }^{12}$ identificaram prevalência elevada de excesso de peso entre crianças de 6 a 10 anos. O estudo foi realizado com 140.878 escolares do Estado de Santa Catarina que tiveram seu estado nutricional classificado segundo critérios do IOTF ${ }^{9}$. As prevalências de sobrepeso e obesidade encontradas foram de $15,4 \%$ e $6,0 \%$, respectivamente.

No município de Piracicaba-SP, pesquisa envolvendo 390 adolescentes com idade entre 10 a 14 anos, revelou prevalência de $21 \%$ de excesso de peso entre os participantes. Neste estudo, a classificação do perfil antropométrico foi realizada com base nos percentis de IMC para a idade, a partir das curvas do $\mathrm{NCHS}^{13}$.

\section{A obesidade e suas conseqüências}

A obesidade pode ser definida de uma maneira simplificada como o acúmulo excessivo de gordura corporal, sob a forma de tecido adiposo, sendo conseqüência de balanço energético positivo, capaz de acarretar prejuízos à saúde dos indivíduos ${ }^{14}$. Sabe-se ainda que a etiologia da obesidade é multifatorial, estando envolvidos em sua gênese tanto aspectos ambientais como genéticos ${ }^{1}$.

O aumento da incidência de obesidade no Brasil é ainda mais preocupante quando se admite que este problema frequentemente não se apresenta de forma isolada, mas como uma condição favorável para a ocorrência de morbidades associadas ${ }^{15,16}$.

Na população adulta, a obesidade aumenta o risco de doença e morte, enquanto na população jovem esse evento vem se convertendo em um problema que causará profundas repercussões na saúde pública ${ }^{17}$.

$\mathrm{O}$ estado nutricional do adolescente é de particular interesse, pois a presença de obesidade nesta faixa etária tem sido associada ao aparecimento precoce de hipertensão arterial, dislipidemias, aumento da ocorrência de diabetes tipo 2, distúrbios na esfera emocional, além de comprometer 
a postura e causar alterações no aparelho locomotor ${ }^{18,19}$.

Os efeitos da obesidade em idade precoce poderão ser notados ainda em longo prazo, tendo sido relatado na literatura um risco de mortalidade aumentado, especialmente por doença coronariana, nos adultos que foram obesos durante a infância e a adolescência ${ }^{20}$.

Além disso, estudos têm demonstrado que a probabilidade de crianças e adolescentes com elevado índice de massa corporal apresentarem excesso de peso aos 35 anos aumenta significativamente à medida que ocorre o aumento da idade. Segundo Guo e Chumlea ${ }^{21}$, a probabilidade de adolescentes que apresentam obesidade aos 18 anos desenvolverem esse distúrbio na vida adulta é de $34 \%$ para os homens e $37 \%$ para as mulheres.

Outra conseqüência importante relacionada à obesidade diz respeito ao custo financeiro elevado que esse distúrbio e suas conseqüências representam para o sistema de saúde e para a sociedade. Os custos diretos com hospitalizações no Brasil indicam que os percentuais despendidos são similares aos de países desenvolvidos ${ }^{22}$.

Estima-se que o custo do tratamento da obesidade corresponda, nos países industrializados, de $2 \%$ a $8 \%$ do gasto total com a saúde. No Brasil, em 2003, segundo o primeiro levantamento sobre os custos da obesidade, aproximadamente 1 bilhão e 100 milhões de reais são gastos a cada ano com internações hospitalares, consultas médicas e remédios para o tratamento do excesso de peso e das doenças associadas. Apenas o Sistema Único de Saúde (SUS) destina 600 milhões de reais para as internações relativas à obesidade. Esse valor equivale a $12 \%$ do que o governo brasileiro despende anualmente com todas as outras doenças ${ }^{23}$.

\section{Obesidade: etiologia multifatorial}

Embora se disponha de um grande acervo de resultados que podem identificar as causas da obesidade, não é tarefa fácil caracterizar sua etiologia. Acredita-se que os determinantes do excesso de peso compõem um complexo conjunto de fatores biológicos, comportamentais e ambientais que se inter-relacionam e se potencializam mutuamente.

Destacam-se entre os fatores relacionados ao sobrepeso e à obesidade características presentes na gestação e no início da vida, como o peso pré-gestacional materno, o fumo durante a gestação e o estado nutricional na infância ${ }^{24,25}$. Entretanto, as mudanças no padrão de alimentação e de atividade física, ocorridas em diversas sociedades, são reconhecidamente os determinantes que mais contribuem para o aumento do excesso de peso ${ }^{26}$.

Oliveira et al. ${ }^{27}$ destacam o papel do desenvolvimento econômico e do processo de urbanização sobre as modificações no estilo de vida da população, traduzidas por padrões alimentares inadequados e modelos de ocupação predominantemente sedentários. As comodidades oferecidas pelo mundo moderno, tais como aparelhos de televisão, telefones sem fio, videogames, computadores, controle remoto, entre outros, têm favorecido a redução do gasto energético.

A adoção de hábitos alimentares conhecidos como "ocidentais", que consistem em uma alimentação rica em gorduras, especialmente de origem animal, açúcar refinado e reduzida ingestão de carboidratos complexos e fibras, tem sido apontada como condição favorecedora para o estoque energético. Além disso, a falta de informação associada à ausência de políticas de saúde que atendam adequadamente a população torna ainda mais grave e preocupante a epidemia de obesidade instalada no país ${ }^{27}$.

\section{Fatores ambientais associados ao sobrepeso e obesidade em adolescentes}

\section{Alimentação}

As práticas alimentares como determinantes diretos do aumento da obesidade têm sido reconhecidas em inúmeros estu$\operatorname{dos}^{28-30}$

Entre os adolescentes, a alimentação 
inadequada, caracterizada pelo consumo excessivo de açúcares simples e gorduras, associada à ingestão insuficiente de frutas e hortaliças, contribui diretamente para o ganho de peso nesse grupo populacional ${ }^{13}$.

Estudo conduzido nos Estados Unidos por Liebman et al..$^{31}$, envolvendo 1.817 adultos, revelou associação positiva entre sobrepeso/obesidade e consumo de bebidas com adição de açúcar, consumo de grandes porções de alimentos, refeições realizadas concomitantemente com outra atividade, e tempo despendido assistindo televisão. Em contrapartida, o aumento do consumo de frutas e hortaliças tem apresentado associação negativa com o ganho de peso ${ }^{32}$.

Segundo Nicklas et al. ${ }^{33}$, as causas dietéticas da obesidade são complexas e ainda pouco estudadas. No entanto, acredita-se que mudanças recentes no padrão alimentar, como maior consumo de refeições fora de casa, aumento do consumo de bebidas adicionadas de açúcar, consumo de porções de alimentos cada vez maiores e freqüência das refeições foram decisivas para a instalação da epidemia de obesidade.

Os referidos autores ressaltam que aproximadamente $46 \%$ dos gastos com alimentação entre as famílias norte-americanas são destinados às refeições fora de casa, sendo $34 \%$ dessa despesa com fast food. Esses números são preocupantes, tendo em vista que o consumo freqüente de refeições fora do domicílio tem apresentado uma associação positiva com o aumento da gordura corporal em adultos ${ }^{34}$. Esta relação pode ser explicada pelos tipos de alimentos consumidos, que frequentemente têm elevada densidade energética, tais como sanduíches, salgados, doces etc.

Atenção especial também deve ser atribuída ao aumento do consumo, por parte dos adolescentes, de bebidas adicionadas de açúcar, como refrigerantes e sucos artificiais. Estudos têm demonstrado ser cada vez mais freqüente a ingestão dessas bebidas pela população jovem, situação que se agravou nas últimas duas décadas do século $\mathrm{XX}^{35}$.

Os resultados de um estudo prospectivo conduzido por Ludwig et al. ${ }^{36}$ mostraram associação positiva entre consumo de bebidas com adição de açúcar e a ocorrência de obesidade em crianças.

De fato, o elevado consumo de bebidas, especialmente refrigerantes, tem sido apontado por pesquisadores como um dos possíveis fatores relacionados ao ganho de peso em vários países ${ }^{37,38}$. Em ampla revisão de literatura realizada por Mourão e Bressan ${ }^{39}$ constatou-se que o estado físico do alimento pode influenciar o consumo alimentar, tanto a curto quanto a longo prazo, e que os alimentos líquidos exercem um menor poder sacietógeno, em comparação com os sólidos. Os possíveis mecanismos envolvidos nesse fraco controle do apetite pelos líquidos são: falta de mastigação, fase cefálica da ingestão menos pronunciada, esvaziamento gástrico mais rápido e fatores cognitivos.

Triches e Giugliani ${ }^{29}$, em estudo transversal do qual participaram 573 crianças de dois municípios da Região Sul do Brasil, verificaram que a omissão do café da manhã e a baixa freqüência do consumo de leite se constituíram em práticas específicas significativamente associadas à obesidade.

Nicklas et al. ${ }^{33}$ argumentam que o hábito de tomar café da manhã pode contribuir para o controle do peso corporal, na medida em que reduz o consumo de gorduras na dieta. A prática regular de desjejum favorece o consumo de grãos, frutas e produtos lácteos. Além disso, o hábito de realizar o desjejum e realizar maior número de refeições durante o dia está relacionado a um padrão mais regular e definido de realização de refeições e está associado, inversamente, ao hábito de "beliscar" alimentos com alta densidade energética ao longo do dia ${ }^{40}$.

O consumo adequado de frutas, legumes e verduras tem sido apontado como um fator protetor para a ocorrência de obesidade. Estudo de caráter transversal conduzido por Oliveira et al..$^{27}$ com 699 crianças de 5 a 9 anos revelou associação inversa entre o consumo de verduras em elevada freqüência (pelo menos 3 vezes por semana) e sobrepeso e obesidade. 
Drapeau et al. ${ }^{41}$ realizaram um estudo prospectivo para verificar o efeito das mudanças nos padrões alimentares sobre o índice de massa corporal. Dentre os resultados mais relevantes, os autores verificaram que a redução do consumo de alimentos com elevado teor de lipídio ou o aumento do consumo de frutas e hortaliças refletiram positivamente no controle do peso corporal. Acredita-se que o efeito protetor desse último grupo no desfecho da obesidade provavelmente se deve à sua baixa densidade energética, elevado conteúdo de fibras e maior poder de saciedade.

\section{Atividade física e sedentarismo}

Embora seja praticamente um consenso que o crescimento da prevalência de excesso de peso seja decorrente do maior consumo de alimentos com elevada densidade energética e especialmente ricos em lipídios e carboidratos simples, esse fato isoladamente não é capaz de explicar o aumento exponencial de obesidade no mundo. De acordo com Flynn et al. ${ }^{42}$, a redução dos níveis de atividade física também parece exercer papel fundamental nesse processo.

Nesse sentido, o maior tempo dedicado às atividades de baixa intensidade, como assistir televisão, usar computador e jogar videogame, tem contribuído para o ganho de peso dos adolescentes.

Evidências confirmam que a prática de atividade física entre jovens apresenta relação inversa com o risco de doenças crônicas não-transmissíveis, dentre elas a obesidade. Além disso, o padrão de atividade na adolescência determina parte dos níveis de atividade física na idade adulta ${ }^{43}$.

Mesmo diante das evidências, estudos revelam que a prevalência de sedentarismo ainda é preocupante, tanto em países desenvolvidos quanto naqueles de renda média e baixa ${ }^{28,44,45}$.

A partir de um estudo de coorte realizado por Hallal et al. ${ }^{46}$ com 4.452 adolescentes com idade entre 10 e 12 anos, verificou-se que $58,2 \%$ da amostra adotavam estilo de vida considerado sedentário (menos de 300 minutos de atividade física/semana).
Oehlschlaeger et al. ${ }^{44}$, estudando a prevalência e os fatores associados ao sedentarismo em uma amostra representativa de 960 adolescentes em Pelotas, RS, encontraram índices de $39 \%$ de sedentarismo.

Nas últimas décadas, observou-se um aumento no número de estudos que investigaram os efeitos da prática de atividades físicas na prevenção de doenças crônicas não-transmissíveis. Entre os benefícios imediatos que a prática de atividades físicas oferece está a melhora na aptidão física relacionada à saúde, como aptidão cardiorrespiratória, força muscular e flexibilidade ${ }^{47}$.

Alguns estudos têm discutido o papel das atividades físicas na prevenção e reabilitação da obesidade entre crianças e adolescentes. Fernandez et al. ${ }^{48}$ observaram que tanto o exercício aeróbico quanto o anaeróbico foram efetivos no tratamento da obesidade. Farias et al. ${ }^{49}$ também verificaram, a partir de um estudo de caso-controle, que a atividade física programada resultou em melhoria na freqüência de sobrepeso e obesidade no grupo que sofreu intervenção.

Entretanto, um trabalho de revisão sistemática revelou que são poucos os estudos longitudinais que mostram que a atividade física atua na prevenção da obesidade entre crianças e adolescentes ${ }^{50}$.

É provável que os resultados divergentes de estudos que relacionam obesidade $\mathrm{e}$ atividade física, se devam às diferenças nos delineamentos das pesquisas, nos períodos de seguimento, nos critérios de classificação do padrão de atividade, entre outros. O fato é que a principal fisiopatologia desse distúrbio é o balanço positivo na ingestão energética. Sendo assim, a adoção de estilos de vida sedentários, que demandam pouca energia, irão contribuir diretamente para o ganho de peso.

Wong et al. ${ }^{51}$ em 1992, já chamavam a atenção para o tempo excessivo dedicado a assistir televisão como sendo um sinal para a identificação de crianças e adolescentes inseridos em estilos de vida que valorizam hábitos alimentares inadequados e inatividade física.

Embora a televisão desempenhe um 
papel relevante na disseminação de informações e cultura, em algumas situações ela pode ser o veículo de mensagens que influenciam negativamente as preferências e escolhas alimentares de crianças e adolescentes, além de desempenhar um efeito direto nos padrões de atividade física ${ }^{52}$.

Goldberg et al..$^{53}$, já em 1978, observaram que crianças expostas a propagandas comerciais frequentemente optavam por alimentos ricos em açúcar, quando comparadas àquelas que não eram expostas a esses anúncios.

Ortega et al. ${ }^{54}$ realizaram um estudo com adolescentes espanhóis com o propósito de analisar as diferenças nos hábitos alimentares e a ingestão de energia e nutrientes como uma função do tempo despendido assistindo televisão. Os resultados deste estudo indicaram que os adolescentes que assistiam TV por mais de 2 horas diariamente consumiam menores quantidades de legumes e frutas quando comparados àqueles que despendiam menos tempo com essa atividade. Observou-se ainda que os indivíduos mais ativos consumiam mais fibras e vitamina $\mathrm{C}$, e apresentaram adequada participação dos macronutrientes no total de energia ingerida.

\section{Considerações finais}

Embora as causas da obesidade sejam de cunho multifatorial, firma-se o reconhecimento de que as mudanças ambientais se constituem nos principais fatores propulsores para o aumento da obesidade, na medida em que estimulam o consumo excessivo de energia combinado a um gasto energético reduzido.

Apesar das limitações na comparação e na análise dos estudos selecionados nesta revisão, os padrões de associação entre determinadas práticas alimentares e de atividade física e o excesso de peso entre os adolescentes mostraram-se consistentes. Por se tratar de fatores passíveis de intervenção, recomenda-se que estratégias de caráter educativo sejam adotadas, tanto em nível coletivo quanto individual, como forma de incentivar o consumo de alimentos saudáveis e estimular a prática de atividade física regular.

A escolha do ambiente escolar para a promoção de hábitos de vida saudáveis também deve ser encorajada, por ser um local de intenso convívio social e propício para atividades educativas.

As intervenções nos hábitos de vida devem ser iniciadas o mais precocemente possível, já que na adolescência ocorrem mudanças importantes na personalidade do indivíduo e por isso é considerada uma fase favorável para a consolidação de hábitos que poderão trazer implicações diretas para a saúde na vida adulta.

\section{Referências}

1. World Health Organization. Obesity: Preventing and Managing the Global Epidemic. Report of a WHO Consultation. Geneva; 2004. WHO Technical Report Series no. 894.

2. Johnson WD, Kroon JJ, Greenway FL, Bouchard C, Ryan D, Katzmarzyk PT. Prevalence of Risk Factors for Metabolic Syndrome in Adolescents: National Health and Nutrition Examination Survey (NHANES), 2001-2006. Prevalence of risk factors for metabolic syndrome in adolescents: National Health and Nutrition Examination Survey (NHANES), 2001-2006. Arch Pediatr Adolesc Med 2009; 163: 371-7.

3. Lee YS. Consequences of childhood obesity. Ann Acad Med Singapore 2009; 38: 75-7.
4. Lobstein T, Baur L, Uauy R. Obesity in children and young people: a crisis in public health. Obes Rev 2004; S5: 4-85.

5. Wang Y, Monteiro CA, Popkin BM. Trends of obesity and underweight in older children and adolescents in the United States, Brazil, China and Russia. Am J Clin Nutr 2002; 74: 971-7.

6. Feijó RB, Sukster EB, Friedrich L, Fialho L, Dziekaniak KS, Christini DW, et al. Estudos de hábitos alimentares em uma amostra de estudantes secundaristas de Porto Alegre. Pediatria 1997; 19(4): 257-62.

7. Neutzling MB, Taddei JAAC, Rodrigues EM, Sigulem DM. Overweight and obesity in Brazilian adolescents. Int J Obes Relat Metab Disord 2000; 24: 869-74. 
8. Terres NG, Pinheiro RT, Horta BL, Pinheiro KAT, Horta LL. Prevalência e fatores associados ao sobrepeso e à obesidade em adolescentes. Rev Saúde Pública 2006; 40(4): 627-33.

9. Cole TJ, Bellizzi MC, Flegal KM, Dietz WH. Establishing a standard definition for child overweight and obesity worldwide: international survey. BMJ 2000; 320(7244): 1240-3.

10. Costa RF, Cintra IP, Fisberg M. Prevalência de sobrepeso e obesidade em escolares da cidade de Santos, SP. Arq Bras Endocrinol Metab 2006; 50: 60-7.

11. Farias ES, Guerra-Junior G, Petroski EL. Estado nutricional de escolares em Porto Velho, Rondônia. Rev Nutr 2008; 21: 401-9.

12. Ricardo GD, Caldeira GV, Corso ACT. Prevalência de sobrepeso e obesidade e indicadores de adiposidade central em escolares de Santa Catarina, Brasil. Rev Bras Epidemiol 2009; 12: 424-35.

13. Toral N, Slater B, Silva MV. Consumo alimentar e excesso de peso de adolescentes de Piracicaba, São Paulo. Rev Nutr 2007; 20(5): 449-59.

14. World Health Organization (WHO). Obesity: preventing and managing the global epidemic. WHO: Geneva; 2000. WHO Technical Report Series, no. 894.

15. Ebbeling CB, Pawlak DB, Ludwig DS Childhood obesity: public-health crisis, common sense cure. Lancet 2002; 360: 473-82.

16. Must A, Spadano J, Coackey EH. The disease burden associated with overweight and obesity. J Am Med Assoc 1999; 282: 1523-9.

17. Moraes AS de, Rosas JB, Mondini L, Freitas ICM de. Prevalência de sobrepeso e obesidade e fatores associados em escolares de área urbana de Chilpancingo, Guerrero, México, 2004. Cad Saúde Pública 2006; 22(6):1289-301.

18. Wright CM, Parker L, Lamont D, Craft AW. Implications of childhood obesity for adult health: Findings from thousand families cohort study. BMJ 2001; 323: 1280-4.

19. Dietz WH. Health consequences of obesity in youth: childhood predictors of adult disease. Pediatrics 1998; 101: 518-25.

20. Must A. Morbidity and mortality associated with elevated body weight in children and adolescents. $\mathrm{Am} \mathrm{J}$ Clin Nutr 1996 S1; 63: 445-7.

21. Guo SS, Chumlea WC. Tracking of body mass index in children in relation to overweight in adulthood. Am J Clin Nutr 1999 S1; 70: 145-8.

22. Sichieri R, Nascimento S, Coutinho W. The burden of hospitalization due to overweight and obesity in Brazil. Cad Saúde Pública 2007; 23: 1721-7.
23. Porto EBS, Morais TW, Raso V. Avaliação do nível de conhecimento multidisciplinar dos futuros profissionais na propedêutica da obesidade. Rev Bras Nutr Emagrecimento 2007; 1(2): 67-71.

24. Marnun AA, Lawlor DA, Alati R, o'Callaghan MJ, Williams, GM, Najman JM. Does maternal smoking during pregnancy have a direct effect on future offspring obesity? Evidence from a prospective birth cohort study. Am J Epidemiol 2006; 164: 317-25.

25. Nelson MC, Gordon-Larsen P, Song Y, Popkin BM. Associations with Adolescent Overweight and Activity Built and Social Environments. Am J Prev Med 2006; 31: 109-17.

26. Popkin BM. The nutrition transition and obesity in the developing world. J Nutr 2001; 131: 871-3.

27. Oliveira AMA de, Cerqueira EMM, Souza JS, Oliveira AC de. Sobrepeso e obesidade infantil: influência de fatores biológicos e ambientais em Feira de Santana, BA. Arq Bras Endocrinol Metab 2003; 47(2): 144-50.

28. Forshee RA, Anderson PA, Storey ML. The role of beverage consumption, physical activity, sedentary behavior, and demographics on body mass index of adolescents. Int J Food Sci Nutr 2004; 55(6): 463-78.

29. Triches RM, Giugliani ERJ. Obesidade, práticas alimentares e conhecimentos de nutrição em escolares. Rev Saúde Pública 2005; 39(4): 541-7.

30. Lima SCVC, Arrais RF, Pedrosa LFC. Avaliação da dieta habitual de crianças e adolescentes com sobrepeso e obesidade. Rev Nutr 2004; 17(4): 469-77.

31. Liebman M, Pelican S, Moore AS, Holmes B, Wardlaw MK, Melcher LM, et al. Dietary intake, eating behavior, and physical activity-related determinants of high body mass index in rural communities in Wyoming, Montana, and Idaho. Int J Obes 2003; 27: 684-92.

32. French SA, Jeffery RW, Murray D. Is Dieting good for you? Prevalence, duration and associated weight and behavior changes for specific weight loss strategies over four years in US adults. Int J Obes Relat Metab Disord 1999; $23: 320-7$.

33. Nicklas TA, Baranowski T, Cullen KW, Berenson G. Eating patterns, dietary quality and obesity. J Am Coll Nutr 2001; 6: 599-608.

34. McCrory MA, Fuss PJ, McCallum JE. Dietary variety within food group: association with energy intake and body fatness in men and women. Am J Clin Nutr 1999; 69:440-7.

35. French SA, Lin BH, Guthrie JF. National trends in soft drink consumption among children and adolescents age 6 to 17 years: prevalence, amounts, and sources, 1977/ 1978 to 1994/1998. JAMA 2003; 103: 1326-31.

36. Ludwig DS, Peterson KE, Gortmaker SL. Relation between consumption of sugar-sweetened drinks and childhood obesity: a prospective, observational analysis. Lancet 2001; 357: 505-8. 
37. Popkin BM, Armstrong LE, Bray GM, Caballero B, Frei $\mathrm{B}$, Willett WC. A new proposed guidance system for beverage consumption in the United States. Am J Clin Nutr 2006; 83(3): 529-42.

38. Vereecken CA, Inchley J, Subramanian SV, Hublet A, Maes L. The relative influence of individual and contextual socio-economic status on consumption of fruit and soft drinks among adolescents in Europe. Eur J Public Health 2005; 15(3): 224-32.

39. Mourão DM, Bressan J. Influência de alimentos líquidos e sólidos no controle do apetite. Rev Nutr 2009; 22: 537 47

40. Kosti RI, Panagiotakos DB, Mihas CC, Alevizos A, Zampelas A, Mariolis A, Tountas Y. Dietary habits, physical activity and prevalence of overweight/obesity among adolescents in Greece: the Vyronas study. Med Sci Monit 2007; 13: 437-44.

41. Drapeau V, Despres JP, Bouchard C, Allard L, Fournier $\mathrm{G}$, Leblanc C, et al. Modifications in food-group consumption are related to long-term body-weight changes. Am J Clin Nutr 2004; 80: 29-37.

42. Flynn MA, McNeil DA, Maloff B, Mutasingwa D, Wu M, Ford C, Tough SC. Reducing obesity and related chronic disease risk in children and youth: a synthesis of evidence with 'best practice' recommendations. Obes Rev 2006; 7(S1): 7-66.

43. Azevedo MR, Araujo CL, Silva MC, Hallal PC. Tracking of physical activity from adolescence to adulthood: a population-based study. Rev Saúde Pública 2007; 41: 6975.

44. Oehlschlaeger MHK, Pinheiro RT, Horta B, Gelatti C, San'Tana P. Prevalência e fatores associados ao sedentarismo em adolescentes de área urbana. Rev Saúde Pública 2004; 38(2): 157-63.

45. Ceschini FL, Florindo AA, Benício MH. Nível de atividade física em adolescentes de uma região de elevado índice de vulnerabilidade juvenil. Rev Bras Cienc Mov 2007; 15: 67-78.
46. Hallal PC, Bertoldi AD, Gonçalves H, Victora CG. Prevalência de sedentarismo e fatores associados em adolescentes de 10-12 anos de idade. Cad Saúde Pública 2006; 22(6): 1277-87.

47. Cooper AR, Wedderkopp N, Wang H, Andersen LB, Froberg K, Page AS. Active travel to school and cardiovascular fitness in Danish children and adolescents. Med Sci Sports Exerc 2006; 38: 1724-31.

48. Fernandez AC, Melo MT, Tufik S, Castro PM, Fisberg M. Influence of aerobic and anaerobic training on the body fat mass in obese adolescents. Rev Bras Med Esporte 2004; 10: 159-64.

49. Farias ES, Paula F, Carvalho WRG, Gonçalves EM, Baldin AD, Guerra-Junior G. Efeito da atividade física programada sobre a composição corporal em escolares adolescentes. J Pediatr 2009; 85: 28-34.

50. Reichert FF, Menezes AMB, Wells JCK, Dumith SC, Hallal PC. Physical activity as a predictor of adolescent body fatness: a systematic review. Sports Med 2009; 39: 279-94.

51. Wong ND, Hei TK, Qaqundah PY, Davidson DM, Bassin SL, Gold KV. Television viewing and pediatric hypercholesterolemia. Pediatrics 1992; 90(1): 75-9.

52. Birch LL, Fisher JO. Development of eating behaviors among children and adolescents. Pediatrics 1998; 101(3): 539-49.

53. Goldberg ME, Gorn GJ, Gibson W. Messages for snack and breakfast food: do they influence children's preferences? J Consumer Res 1978; 5: 73-81.

54. Ortega EM, Andrés P, Requejo AM, López-Sobaler AM, Redondo MR, González-Fernández M. Influence of the time spent watching television on the dietary habits, energy intake and nutrient intake of a group of Spanish adolescents. Nutr Res 1996; 16(9): 1467-70.

Recebido em: 15/06/09

Versão final reapresentada em: 24/02/10 Aprovado em: 28/02/10 